\title{
23. COMMISSION DE LA CARTE DU CIEL
}

\section{Compte-rendu de la Séance du 26 Août 1964}

PrÉsIdent: P. Sémirot.

SECRÉTAIRE: W. J. Luyten.

Constitution de la Commission 23 pour la période 1964-1967

Président: P. Sémirot.

Vice-Président: G. J. Järnefelt.

Comité d'organisation: P. Couderc, A. N. Deutsch, F. Fernandez de la Puente, H. Kox, W. J. Luyten, H. W. Wood.

Membres: Abhyankar, Bouigue, Danjon, Eichhorn, Fracastoro, Harris, Landi Dessy, Lourens, O'Connell, Paloque, Warzée.

\section{Compte-Rendu Financier}

Les subventions accordées par l'UAI aux observatoires de Catane, Paris, Sydney, Tacubaya, ont permis l'achèvement de l'impression des catalogues photographiques de Paris-Uccle, de Melbourne, de Tacubaya et la réimpression des catalogues de Catane. La Commission 23 dispose d'un excédent de recettes s'élevant à \$2 I65.9r.

Le budget de la Commission 23 est approuvé à l'unaminité.

\section{Draft Report}

Le Draft Report est adopté sans modification. La discussion des questions mises à l'ordre du jour est alors abordée.

\section{Questions I et 2: Calcul des constantes}

L'impression du catalogue photographique est maintenant terminée. P. Sémirot renouvelle ses remerciements à Mme Paris Pishmish et MM. Couderc, Fracastoro, Wood.

Les astronomes possèdent donc les coordonnées rectilignes mesurées $x, y$ de toutes les étoiles du catalogue. Cette connaissance est cependant insuffisante et le catalogue ne peut être utile que si les constantes sont calculées. Les positions des étoiles repères pour l'instant de la prise des clichés seront calculées à partir des positions et des mouvements propres déduits de l'AGK2 et de l'AGK 3 . A partir des coordonnées équatoriales ainsi obtenues, des constantes de rère approximation pourront être calculées. A partir de ces constantes il sera possible de recalculer les $\alpha, \delta$ des étoiles repères et de redéterminer les mouvements propres de ces étoiles. Il est bien évident que les positions $\alpha, \delta$ extraites du catalogue ne seront pas indépendantes des positions $\mathrm{AGK}_{2}$ et $\mathrm{AGK}_{3}$, mais les mouvements propres des étoiles de l'AGK seront cependant améliorés. Dieckvoss et Kox ont trouvé que par cette méthode de calcul les erreurs moyennes évaluées à $0^{\prime \prime} \cdot 008$ par an ont diminué et sont devenues égales à o"·004 par an.

Les positions des images des étoiles sur une plaque photographique peuvent être affectées d'erreurs énumérées dans le Draft Report. Couderc a étudié l'effet de magnitude $m$ et l'effet de distance image-centre de la plaque, soit $r$, dans le cas particulier de clichés obtenus suivant la méthode dite de raccordement imaginée par Turner. Son étude lui a permis de trouver une relation permettant la correction des coordonnées rectilignes $x, y$ en fonction de $r$ et de $m$. Couderc signale que, dans sa thèse, il a également discuté le catalogue de raccordement de 
Paris. Couderc dit que chaque observatoire pourrait également établir une formule permettant le calcul des corrections. Sémirot pense qu'il ne peut pas y avoir de formule unique pour toute une zone de catalogue et que les corrections doivent être calculées pour chaque cliché. Il semble que l'une ou l'autre des méthodes de calcul puisse être adoptée:

(I) Calculer les constantes en incluant les termes correctifs dans les équations. Dieckvoss dit que le nombre des étoiles repères, une vingtaine par cliché, est peut-être trop faible pour permettre ce procédé.

(2) Calculer les constantes sans inclure les termes correctifs et déterminer ceux-ci par l'étude des résidus. En tenant compte des résultats obtenus il faudrait procéder à un nouveau calcul des constantes.

Pour la zone de Paris étudiée à Bergedorf, Dieckvoss n'a pas trouvé de termes correctifs sensibles.

Wood fait remarquer que, pour la zone Sud, il n'y a pas l'équivalent de l'AGK3.

Murray se demande s'il est nécessaire de calculer les constantes. Il est presque invraisemblable, dit-il, que les constantes de toutes les plaques soient utilisées. Il pense qu'il serait plus judicieux de calculer les coordonnées standard $X, Y$ de toutes les étoiles de l'AGK2, de l'AGK $_{3}$, de Yale et de CPC en choisissant pour centres les centres des clichés du catalogue photographique et de publier en même temps les termes $\mu_{\alpha} \cos \delta, \mu_{\delta}$ permettant le calcul immédiat des quantités $\Delta X, \Delta Y$ dues aux mouvements propres en $\alpha$ et en $\delta$. Le 'Catalogue de coordonnées standard' ainsi formé permettrait à chaque astronome de calculer les constantes des plaques qu'il aurait à utiliser.

Pour calculer les constantes des plaques, il est nécessaire de posséder les coordonnées équatoriales des étoiles repères et leur mouvement propre. A cet effet, il faut connaître pour chaque étoile les coordonnées équatoriales déterminées à des époques différentes. La question 2 du Draft Report était celle-ci: faut-il pour chaque étoile repère inscrire sur carte perforée les coordonnées équatoriales observées à des époques différentes? Dieckvoss fait connaître qu'il a déjà $4 \times 10^{5}$ cartes et que l'on a pu calculer pour l'AGK2 de Bonn et de Bergedorf les corrections dues aux erreurs introduites par les systèmes optiques et aux erreurs de mesure. Hors séance, Herget fait savoir que, pour la zone de Bordeaux, une carte perforée correspondant à chaque étoile repère contient les coordonnées équatoriales de l'AGK1, de l'AGK2, de Yale, du catalogue $\mathrm{S}$ de Bordeaux ainsi que ses coordonnées rectilignes $x, y$. Le travail est terminé et sera mis sur bande magnétique par Scott.

Conclusion. La discussion sur les questions I et 2 étant close, la Commission approuve le calcul des constantes de tous les clichés du catalogue à l'exception des zones photographiées récemment ou qui pourraient présenter quelque anomalie. Couderc propose que les constantes des clichés de l'hémisphère Nord soient calculées à Hambourg, mais Dieckvoss fait remarquer que la machine à calculer électronique n'est à sa disposition que pendant quelques heures par semaine. Des centres possédant des machines pourraient donc collaborer avec Hambourg. Bordeaux, Paris, Toulouse proposent leur concours pour perforer les cartes avec les coordonnées exactes du catalogue photographique après le calcul automatique des coordonnées approximatives à partir des données de l'AGK2 et de l'AGK3.

Eichhorn a offert son concours pour participer aux nouvelles réductions d'une grande partie des clichés du catalogue photographique.

Couderc propose qu'une subvention soit accordée à l'Observatoire de Hambourg et, malgré le mauvais état de ses finances, l'UAI a bien voulu attribuer $\$ 2000$ à la Commission 23.

\section{Questions 3 et 4}

Murray et les membres de la Commission jugent inutile de remesurer les clichés non 
mesurés avec des machines à vis. Il est jugé également inutile de reprendre les clichés du catalogue et de la carte qui ont disparu ou sont détériorés.

\section{Questions 5 et 6}

Après intervention de Murray, la Commission est d'accord pour que les mouvements propres de toutes les étoiles du catalogue ne soient pas mesurés. Seuls les mouvements propres d'étoiles particulières doivent être déterminés, par exemple les étoiles de la liste de Plaut et de Van Herk. Un programme important est celui de la détermination des mouvements propres des étoiles des Selected Areas, spécialement ceux des étoiles de basse latitude galactique. Vasilevskis propose une réunion des Commissions 23 et 24 et du Comité des 'Selected Area' pour établir un programme d'observations particulièrement importantes pour les étoiles faibles. Une circulaire ultérieure mettra les membres de la Commission au courant des décisions adoptées.

Murray fait part du projet d'Eichhorn consistant en la réobservation des étoiles avec des télescopes de type Baker. Avant de prendre une décision, il serait nécessaire d'étudier le champ des plaques du 'Palomar Sky Survey' par les méthodes d'Eichhorn. Murray dit que Dixon a montré que les petites caméras de Schmidt peuvent être utilisées. Un tel projet n'est pas de la compétence de la Commission de la Carte du Ciel. Van Herk présente quelques remarques sur les mouvements propres ultérieurs de l'AGK3.

\section{Question 7}

Vasilevskis dit que la machine à mesurer automatique présente les avantages suivants: précision, rapidité et obtention rapide des résultats pour l'astronome calculateur. L'Observatoire de Lick espère inaugurer la machine vers la fin de l'année 1964.

En ce qui concerne le guidage automatique des équatoriaux, Vasilevskis montre que suivant le type d'équatorial utilisé les méthodes automatiques de guidage sont différentes. A Lick, on guide en $x$ et $y$, alors qu'avec les astrographes on guide en $\alpha$ et $\delta$. Dans ce dernier cas, le terme en $\sec \delta$ ' introduit et il est parfois difficile de trouver dans la région du ciel à photographier une étoile brillante.

\section{Question 8: Etoiles doubles, mouvement propre de chacune des composantes d'un couple}

Osvald, du Leander McCormick Observatory, a fait connaître, par une lettre, qu'il détermine les mouvements propres des étoiles doubles serrées, et, pour mener à bien son travail, il désirerait le calcul des constantes des clichés du catalogue photographique.

Dommanget croit que les observateurs d'étoiles doubles pourraient apporter une contribution intéressante à la détermination des mouvements propres. Des couples séparés de plusieurs secondes d'arc ont été observés il y a plus d'un siècle et pour certains d'entre eux les observations ont été poursuivies jusqu'à maintenant. Une trajectoire relative rectiligne peut alors être calculée avec une précision qui atteint au moins celle désirée pour les mouvements propres. La connaissance de cette trajectoire peut donc être de grande utilité soit que l'on désire connaître le mouvement propre du compagnon connaissant celui de l'étoile principale soit que l'on désire ajuster l'un vis-à-vis de l'autre le mouvement propre connu pour chacune des composantes en se servant de leur mouvement relatif. Dommanget trouve qu'en général les valeurs publiées dans le 'Eigenbewegungs-Lexicon' sont plus précises que celles des catalogues de Yale.

La discussion sur les voeux et questions à débattre est close et une mise au point est faite sur les travaux entrepris depuis la réunion de Berkeley. Aucune remarque n'est présentée sur les paragraphes suivants du Draft Report: mouvements propres, rattachement aux galaxies, position de la Lune et Carte du Ciel. Deutsch annonce que l'Observatoire de Bordeaux a terminé le travail qui lui avait été confié. 
Organisation du calcul des constantes

Au cours de la réunion du 26 Août, il a été convenu que Sémirot s'entendrait avec Dieckvoss pour fixer les crédits à demander à l'UAI et organiser le calcul des constantes. Les astronomes suivants ont assisté à cet entretien: Lacroute, Couderc, Sémirot, Dieckvoss, Kox, Günther, C. F. Martin, Mlle Canavaggia. Le compte-rendu de cette réunion qui a eu lieu le 2 Septembre a été joint au compte-rendu de la réunion du 26 Août afin d'abréger la rédaction et pour rendre plus claire la discussion des travaux de la Commission 23. Il a été décidé de verser tous les crédits de la Commission 23 à l'Observatoire de Bergedorf qui fixera la méthode à suivre pour le calcul des constantes et répartira le travail de calcul entre les observatoires de Bergedorf, Bordeaux, Paris, Toulouse. Eichhorn von Wurmb participera également à ces calculs. 